\title{
Irregular Generic Detection Aided Iterative Downlink SDMA Systems
}

\author{
Chun-Yi Wei and Lajos Hanzo \\ School of ECS., Univ. of Southampton, SO17 1BJ, UK. \\ Tel: +44-23-80-593 125, Fax: +44-23-80-593 045 \\ Email: lh@ecs.soton.ac.uk, http://www-mobile.ecs.soton.ac.uk
}

\begin{abstract}
When an iterative-decoding aided system is configured to operate at a near-capacity performance, an excessive complexity may be imposed by the iterative process. In this paper, we propose the novel framework of generic detection, which invokes appropriately amalgamated multiple detectors. Hence the proposed Irregular Generic Detection (IrGD) algorithm may reduce the complexity of iterative detectors. We show in the context of an iterative DownLink (DL) Space Division Multiple Access (SDMA) system that the proposed IrGD may indeed reduce the complexity of the iterative receiver. The IrGD aided DL-SDMA system detects the appropriate fractions of the received bitstream with the aid of different detectors. This allows us to match the Extrinsic Information Transfer (EXIT) curve of the detector to that of the channel decoder, hence facilitating a near-capacity operation, which reducing the detection complexity by about $28 \%$ compared to a powerful near-Maximum-Likelihood (ML) sphere detector benchmark system.
\end{abstract}

\section{INTRODUCTION}

Iterative detection aided systems may dramatically improve the attainable system performance and often result in a near-capacity performance [1]. However, this may impose an increased complexity. As an extension of [2], we introduce the complexityconscious frame work of a generic hybrid detection algorithm, which is optimized using Extrinsic Information Transfer (EXIT) charts [3].

In order to reduce the overall iterative decoding complexity, we take into account both the complexity per iterative detection stage and the number of iterations required. We observed with the aid of ten Brink's EXIT charts [3] that the different detectors require different numbers of iterations for reaching the $\left(I_{A}, I_{E}\right)=(1,1)$ point of the EXIT chart, provided that the $E_{b} / N_{0}$ value experienced is sufficiently high for maintaining an open EXIT tunnel. Specifically, more powerful detectors, such as the Maximum A Posteriori probability (MAP) and the Optimized Hierarchy Reduced Search Algorithm (OHRSA) aided detector of [4], [5] require a lower number of iterations. At the same time, the family of less powerful detectors, such as the Minimum Mean Square Error (MMSE) based [6], necessitate a higher number of iterations. At higher $E_{b} / N_{0}$ values the resultant EXIT-tunnel tends to be more widely open and hence the system tends to require a lower number of iterations to reach the point of perfect convergence at $\left(I_{A}, I_{E}\right)=(1,1)$, where typically an infinitesimally low BER is achieved. By contrast, at lower $E_{b} / N_{0}$ values the system tends to require a higher number of iterations to attain an infinitesimally low BER. Therefore, the complexity of iterative decoding depends on the complexity of the detector as well as on the $E_{b} / N_{0}$ level encountered. In order to reduce the complexity imposed by the iterative receiver of [2], we will propose a novel detection algorithm, which we refer to

The financial support of the EU under the auspices of the Optimix project, as well as that the EPSRC UK is gratefully acknowledged. as the Irregular Generic Detection (IrGD) algorithm, where the terminology 'irregular' will be justified later.

The philosophy of the IrGD is that of utilizing multiple detectors in the iterative decoding process, instead of a single detector. When invoking multiple detectors applied to different appropriately selected fractions of the input bit-stream, the complexity of the IrGD may be quantified by weighting the complexity of each constituent detector according to their 'duty cycle', which is defined here the aforementioned relative fraction of the input bit-stream. Designing a IrGD requires searching for the lowest-complexity 'optimum' duty cycle of the candidate detectors, namely that which has the lowest total complexity for the entire iterative decoding process and hence we refer to this as the optimization of the IrGD scheme. The details of the IrGD scheme's optimization will be discussed in Section V.

The proposed IrGD algorithm has the following attractive properties:

1) By invoking appropriately amalgamated multiple detectors, the complexity of the IrGD becomes tunable, since it is obtained by weighting the complexity of the individual detectors invoked according to their duty cycle.

2) The design of the IrGD scheme may be readily accomplished with the aid of EXIT chart. The EXIT function of the IrGD aided system and its convergence behaviour can also be readily predicted and analyzed. In other words, the design of the IrGD arrangement can be simplified to an inner-EXIT curve fitting or matching problem, adopting a philosophy originally suggested for the design of nearcapacity IrRegular Convolutional Codes (IRCC) [7]. This conceptual similarity between the IRCCs [7] and the IrGDs justified our choice of terminology.

3) Similarly to IRCCs, the design of the IrGD requires finding the specific weighting coefficients for the various IrGD receiver components, which results in the lowest total complexity. This requires an 'off-line' optimization. Hence its complexity does not contribute to the complexity of the real-time iterative decoding process.

The rest of this paper is structured as follows. First, we introduce the IrGD algorithm in more depth in Section II. In Section III, we illustrate the structure of the IrGD aided iterative DL-SDMA system considered and the constituent detectors of the IrGD in Section IV, followed by the details of the weighting factor optimization process applied to our iterative DL-SDMA system in Section V. In Section VI, we analyze the convergence of our iterative DL-SDMA scheme with the aid of EXIT charts. The corresponding performance results are detailed in Section VII, followed by our conclusions in Section VIII. 


\section{IrRegular Generic Detection Algorithm}

The philosophy of the IrGD technique is that of utilizing different candidate detectors for detecting predetermined segments of the transmitted signals, instead of using a single detector, as justified later in this section. Based on the appropriately optimized fractions of the received signal, each detector generates its soft-bit estimates of the appropriate fractions of the transmitted signals. More specifically, for a transmission block containing $L$ encoded bits and assuming that $N_{b p s}$ bits per symbol are transmitted by the modulator, we have $L / N_{b p s}$ symbols per transmission block, where each transmission block is mapped to a separate time-slot. At the receiver, we invoke the proposed IrGD scheme, which is constituted by $N_{\text {det }}$ component detectors. Let $C_{j}$ be the complexity of the $j$-th component detector of the IrGD scheme. Then the weighting coefficient $\alpha_{j}$ has to satisfy:

$$
1=\sum_{j=1}^{N_{\text {det }}} \alpha_{j}, C_{g d}=\sum_{j=1}^{N_{\text {det }}} \alpha_{j} C_{j}, \text { and } \alpha_{j} \in[0,1], \forall j .
$$

where $C_{g d}$ is the average complexity of the IrGD scheme.

Furthermore, each constituent detector generates $\alpha_{j} L$ soft-bits for a transmission block containing $L$ bits. The IrGD's design philosophy is that it allows us to superimpose the EXIT curves of the individual component detectors appropriately weighted by the optimum $\alpha_{j}$ value, which facilitates the matching of the detector's EXIT curve to that of the outer channel decoder. It was shown in [7] that this EXIT-curve matching has the potential of minimizing the open EXIT-tunnel's area and hence it is capable of a near-capacity operation, while maintaining an infinitesimally low BER. To elaborate a little further, according to [7], the EXIT function $T_{g d}\left(I_{i n}\right)$, which characterizes the detector referred to synonymously as the inner 'decoder' of the IrGD aided system, is given by

$$
T_{g d}\left(I_{i n}\right)=\sum_{j=1}^{N_{d e t}} \alpha_{j} T_{j}\left(I_{i n}\right),
$$

where $T_{j}\left(I_{i n}\right)$ is the EXIT function of the $j$-th component detector invoked by the IrGD scheme.

In summary, using the weighting-coefficient vector $\alpha$ formed by the weighting coefficients $\alpha_{j}$ for $j=1,2, \cdots, N_{\text {det }}$ satisfying Equation 1 creates a IrGD having a given average computational complexity of $C_{g d}$. Given the weighting-coefficient vector $\boldsymbol{\alpha}$, the EXIT function of $T_{g d}\left(I_{i n}\right)$, characterizing the inner decoder's EXIT curve can be generated based on Equation 2. Therefore, we can design the superimposed EXIT function $T_{g d}\left(I_{i n}\right)$ of the IrGD aided system by optimizing the weightingcoefficient vector $\boldsymbol{\alpha}$, for the sake of minimizing the open EXITtunnel area and hence to facilitate near-capacity operation, while maintaining an infinitesimally low BER.

In the next section, we will apply the IrGD in the context of an iterative DL-SDMA system in [2].

\section{SYSTEM MODEL}

Based on the iterative DL-SDMA system of [2], the structure of the system considered is depicted in Figure 1. The BaseStation (BS) employs $M$ transmit antennas for supporting $K$ Mobile Stations (MSs), where each of the MSs employs $N_{k}$ receive antennas. We assume a flat-fading Multi-Input and MultiOutput (MIMO) channel. Let $\mathbf{s}^{(k)} \in \mathbb{C}^{L_{k} \times 1}$ be a complexvalued column vector, which denotes the data symbol vector to

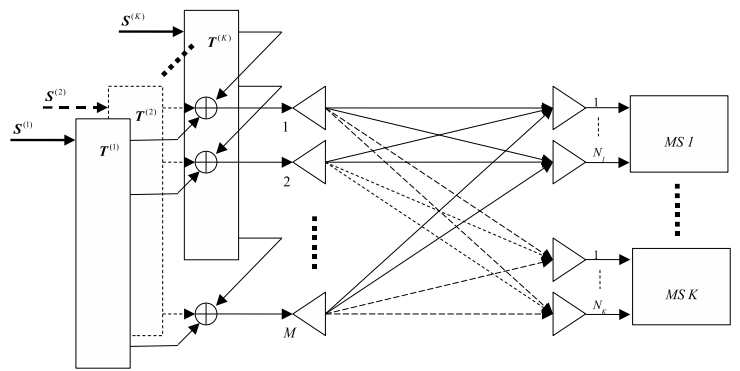

Fig. 1. Multiuser transmission in the DL-SDMA system

be transmitted to the $k$-th MS, while $L_{k}$ represents the number of independent data symbols contained in $\mathbf{s}^{(k)}$. Additionally, we define the so-called Space-Time Preprocessor (STP) matrix $\mathbf{T}^{(k)} \in \mathbb{C}^{M \times L_{k}}$, which was designed for the sake of eliminating the MUI [8] as detailed in [2].

As illustrated in Figure 2, the data bits are encoded by both the channel encoder and the Unity-Rate Coder (URC), which is constituted by a convolutional encoder using a single shift register stage [9]. The three parallel paths in Figure 2 indicate that not only the IrGD, but also the transmitter has to process the fraction of $\alpha_{1} L, \alpha_{2} L$ and $\alpha_{3} L$ bits separately, although the same URC and modulation schemes are employed. The weightingcoefficient vector $\boldsymbol{\alpha}$ is used for partitioning the coded bits into the appropriate-length segments for the URC encoders. For example, for a transmission block containing $L$ encoded bits, the $j$-th URC encoder encodes $\alpha_{j} L$ bits. Assuming that $N_{b p s}$ bits per symbol are used for transmission, $\alpha_{j} L / N_{b p s}$ modulated symbols are generated by the $j$-th modulator. Both the number $N_{d e t}$ and the weight of the constituent detectors used for creating the IrGD may be chosen to minimize the complexity of the IrGD while maintaining the target BER. As an illustration, in this study, we utilize three detectors $\left(N_{\text {det }}=3\right)$ to create the IrGD, as detailed in Section IV.

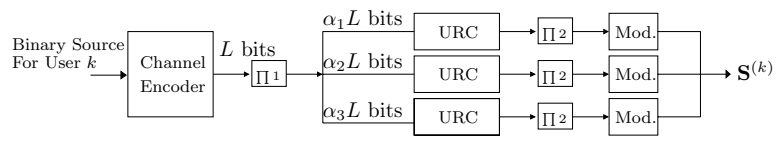

Fig. 2. Generating the precoded data symbols for the $k$-th user

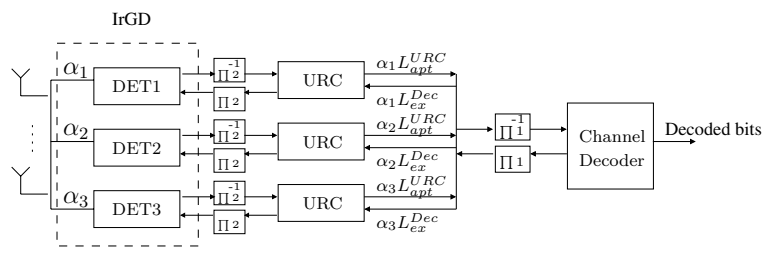

Fig. 3. The structure of the receiver of the IrGD aided iterative DLSDMA system

Furthermore, Figure 3 illustrates the structure of the MS's receivers. As shown in Figure 3, the IrGD is assembled from three different detectors. The received signals are partitioned into appropriate-length segments according to the weightingcoefficient vector $\alpha$. Each detector then processes the corresponding fraction of the received signal. The $j$-th URC decoder generates $\alpha_{j} L_{a p t}^{U R C}$ number of soft-bits for the channel decoder. 
1 Then iterative detection is invoked for exchanging extrinsic information between the constituent detectors, URC decoders and the channel decoder. The extrinsic information bits generated by the channel decoder are also partitioned into appropriate-length segments according to the weighting-coefficient vector $\boldsymbol{\alpha}$ and then they are fed back to the URC decoders.

As a simplifying assumption, the perfect channel knowledge is assumed to be available at both the transmitter and receiver side in this study. Therefore the MUI may be entirely eliminated [2]. In this case, the system can be considered as a number of parallel single-user MIMO systems. In this paper, we focus our attention on introducing the IrGD algorithm itself. The impact of imperfect channel knowledge will be considered in our future studies.

\section{The Constituent Detectors}

We use the following linear and nonlinear detectors for constructing the IrGD:

1) Log Maximum A Posteriori Probability (Log-MAP) detector [6];

2) Optimized Hierarchy Reduced Search Algorithm (OHRSA) aided detector [5], which is a reducedcomplexity near-ML sphere detector;

3) Minimum Mean Square Error (MMSE) detector [6], which has the lowest complexity;

Any of these detectors can be considered as a stand-alone SDMA detector, which were detailed in [4], [6]. All of these detectors are capable of generating soft-bit LLR information for supporting iterative detection.

While amalgamating these detectors into a IrGD, the resultant computational complexity of the IrGD arrangement may also be controlled by appropriately designing the weighting-coefficient vector $\boldsymbol{\alpha}$. In Table I, we summarize the average computational complexity of each component detector, which was quantified in term of the number of multiplications and additions required for generating the LLR of each soft-bit. Given Equation 1, the associated computational complexity of the IrGD may be characterized by taking the computational complexity of the individual component detectors of Table I into account. As stated in [5], the direct calculation of the OHRSA detector's computational complexity is infeasible, since it is SNR-dependent. Hence the complexity of the OHRSA detector was compared to that of the other detectors using the computer simulations illustrated in [5].

\section{Optimization of the IRREgUlar Generic Detector}

The weighting-coefficient vector $\boldsymbol{\alpha} \in \mathbb{R}^{N_{\text {det }}}$ has to satisfy Equation 1 and Equation 2. Let $\mathcal{C}$ be a set containing all the candidate solutions $\boldsymbol{\alpha}$. We may find the weighting-coefficient vector $\alpha \in \mathcal{C}$ by conducting a search similar to that suggested in [7]. By defining a $\left(N_{p} \times N_{\text {det }}\right)$-element matrix $\boldsymbol{A}$, which is constituted by $N_{d e t}$ number of EXIT functions $T_{j}\left(I_{i n}\right), j=1, \cdots, N_{\text {det }}$, and a vector $\boldsymbol{b}=T_{c c}^{-1}\left(I_{i n}\right)$, for $I_{i n} \in\left\{i_{1}, i_{2}, \cdots, i_{N_{p}}\right\}$, where

${ }^{1} L_{a p t}^{U R C}$ is the number of the soft-bits, which are represented by the Log Likelihood Ratio (LLR) [6] values generated by the URC as the a posteriori information. $L_{e x}^{D e c}$ is the number of the extrinsic soft-bits generated by the channel decoder.
$N_{p}$ is the number of EXIT functions, we have

$$
\mathbf{A}=\left[\begin{array}{cccc}
T_{1}\left(i_{1}\right) & T_{2}\left(i_{1}\right) & \ldots & T_{N_{\text {det }}}\left(i_{1}\right) \\
T_{1}\left(i_{2}\right) & T_{2}\left(i_{2}\right) & \cdots & T_{N_{\text {det }}}\left(i_{2}\right) \\
\vdots & \vdots & \ldots & \vdots \\
T_{1}\left(i_{N_{p}}\right) & T_{2}\left(i_{N_{p}}\right) & \cdots & T_{N_{\text {det }}}\left(i_{N_{p}}\right)
\end{array}\right]
$$

and

$$
\boldsymbol{b}=\left[\begin{array}{c}
T_{c c, 1}^{-1}\left(i_{1}\right) \\
T_{c c, 1}^{-1}\left(i_{2}\right) \\
\vdots \\
T_{c c, 1}^{-1}\left(i_{N_{p}}\right)
\end{array}\right]
$$

Our Objective Function (OF) $J(\boldsymbol{\alpha})$ may be defined as

$$
J(\boldsymbol{\alpha})=\|\mathbf{A} \boldsymbol{\alpha}-\boldsymbol{b}\|^{2},
$$

which represents the area between the outer channel decoder's EXIT curve and the inner decoder's EXIT curve, where the latter is constituted by the combined IrGD and URC decoder. Naturally, all elements of $e$, where we have $e=(\mathbf{A} \alpha-b)$, have to be larger than zero, since they physically represent the area between the outer and inner decoder's EXIT curve. We define a subset $\mathcal{A} \subset \mathcal{C}$ containing all weighting coefficient vectors $\boldsymbol{\alpha}$ meeting this constraint. Secondly, for a specific fixed computational complexity $C_{g d}$, we opt for that particular $\boldsymbol{\alpha}$ value, which is associated with a higher area $\mathrm{J}(\boldsymbol{\alpha})$ between the outer EXIT curve $T_{c c}^{-1}\left(I_{i n}\right)$ and the inner EXIT curve $T_{g d}\left(I_{i n}\right)$, which implies requiring a low number of decoding iterations. Therefore, we assume that the optimal weighting-coefficient vector $\boldsymbol{\alpha}_{\text {opt }}$ can be obtained by finding the $\boldsymbol{\alpha}$ value satisfying:

$$
\boldsymbol{\alpha}_{o p t}=\max _{\boldsymbol{\alpha} \in \mathcal{A}} J(\boldsymbol{\alpha}) .
$$

\section{IRGD DESIGN AND ANALYSIS USING EXIT CHARTS}

In this section, we will demonstrate how EXIT charts may be used to assist us in the design of the IrGD and in the analysis of the systems' iterative decoding performance.

Let us first define the IrGD's per-iteration target complexity ratio $r_{g d}$ with respect to the Log-MAP detector's complexity $C_{L o g-M A P}$ as follows:

$$
r_{g d}=C_{g d} / C_{L o g-M A P}
$$

where again $C_{g d}$ is the computational complexity of the IrGD.

Furthermore, the total complexity $C_{g d, i t e r}{ }^{2}$ of the IrGD embedded into the iterative decoding process, is given by

$$
C_{g d, i t e r}=C_{g d} \times I_{i t e r, g d},
$$

where $I_{i t e r, g d}$ is the number of IrGD iterations required for achieving convergence to the $\left(I_{A}, I_{E}\right)=(1,1)$ point in the EXIT chart. Therefore, the total complexity ratio of the IrGD is given by

$$
r_{g d, i t e r}=C_{g d, i t e r} / C_{L o g-M A P, i t e r},
$$

where $C_{L o g-M A P, i t e r}$ is the total complexity imposed by the iterative Log-MAP detector, when attaining perfect convergence to the $\left(I_{A}, I_{E}\right)=(1,1)$ point in the EXIT chart.

In Figure 4, we illustrate a IrGD design example for $r_{g d}=$ 0.4 , configured for operation at $E_{b} / N_{0}=1.7 d B$. The inner

\footnotetext{
${ }^{2}$ The total complexity is defined here as the product of the per-iteration
} complexity and the number of iterations required for approaching an infinitesimally low BER. 
TABLE I

THE AVERAGE COMPUTATIONAL COMPLEXITY OF COMPONENT DETECTORS ON THE BASIS OF GENERATING THE LLR OF EACH SOFT-BIT

\begin{tabular}{|c|c|}
\hline $\mathcal{C}_{\text {Log-MAP }}$ & $2 \cdot\left[2^{L_{k}} \log _{2} \mathcal{M}_{c-1} \cdot\left(3 N_{k}+2 N_{k} L_{k}\right)\right]$, where $\mathcal{M}_{c}$ is the constellation size of the modulation scheme used. \\
\hline $\mathcal{C}_{\text {OHRSA }}$ & calculated using computer simulations as illustrated in [5] \\
\hline $\mathcal{C}_{\text {MMSE }}$ & {$\left[L_{k}{ }^{3}+2\left(L_{k} N_{k}^{2}+L_{k}^{2} N_{k}+L_{k} N_{k}+L_{k}\right)\right] /\left(L_{k} \log _{2} \mathcal{M}_{c}\right)+\left(2 N_{k}+3\right)$} \\
\hline
\end{tabular}

\begin{tabular}{|l|r|}
\hline $\begin{array}{l}\text { Channel Coder } \\
\text { Interleaver length }\end{array}$ & $\begin{array}{r}\text { rate-0.5 RSC [5,7] } \\
10^{5} \text { bits }\end{array}$ \\
\hline Modulation & 4QAM \\
\hline Number of users & $K=3$ \\
\hline Number of transmit antennas & $M=6$ \\
\hline $\begin{array}{l}\text { Number of independent data streams } \\
\text { transmitted for the } k \text {-th user }\end{array}$ & $L_{k}=2$, for $k=1,2,3$. \\
\hline Number of receive antennas & $N_{k}=2$, for $k=1,2,3$. \\
\hline
\end{tabular}

\begin{tabular}{|l|l|c|r|l|}
\hline$r_{g d}$ & $\boldsymbol{\alpha}$ & $I_{g d}$ & $C_{g d, i t e r}$ & $r_{g d, i t e r}$ \\
\hline 1 & {$[0 ; 0 ; 1]$} & 5 & 2240.00 & 1.0 \\
\hline 0.5 & {$[0 ; 0.957 ; 0.043]$} & 5 & 1120.00 & 0.5 \\
\hline 0.4 & {$[0.27 ; 0.73 ; 0]$} & 7 & 1254.40 & 0.56 \\
\hline 0.3 & {$[0.61 ; 0.39 ; 0]$} & 8 & 1075.20 & 0.48 \\
\hline 0.21 & {$[0.92246 ; 0.07754 ; 0]$} & 9 & 846.72 & 0.378 \\
\hline 0.2 & {$[0.957 ; 0.043 ; 0]$} & 9 & 806.40 & 0.36 \\
\hline 0.19 & {$[0.992 ; 0.008 ; 0]$} & 10 & 851.20 & 0.38 \\
\hline 0.18 & N/A & N/A & N/A & N/A \\
\hline
\end{tabular}

EXIT curve $T_{g d}\left(I_{i n}\right)$ of the IrGD aided iterative DL-SDMA system using $\boldsymbol{\alpha}=[0.27 ; 0.73 ; 0]$ is shown as the solid line in Figure 4. According to Equation 2, the inner EXIT curve of the IrGD aided iterative DL-SDMA system is constituted by a linear combination of the inner EXIT curves of the iterative DL-SDMA system employing the MMSE, OHRSA and Log-MAP detectors. Recall that $\boldsymbol{\alpha}$ determines the percentage of soft-bits decoded by each constituted detector and in this case only the MMSE and OHRSA detectors were employed, where the MMSE detector had a duty cycle of $27 \%$, while the OHRSA detector a duty cycle of $73 \%$. The elements in $\alpha=[0.27 ; 0.73 ; 0]$ represent the duty cycles of the MMSE, OHRSA and Log-MAP detectors in this order.

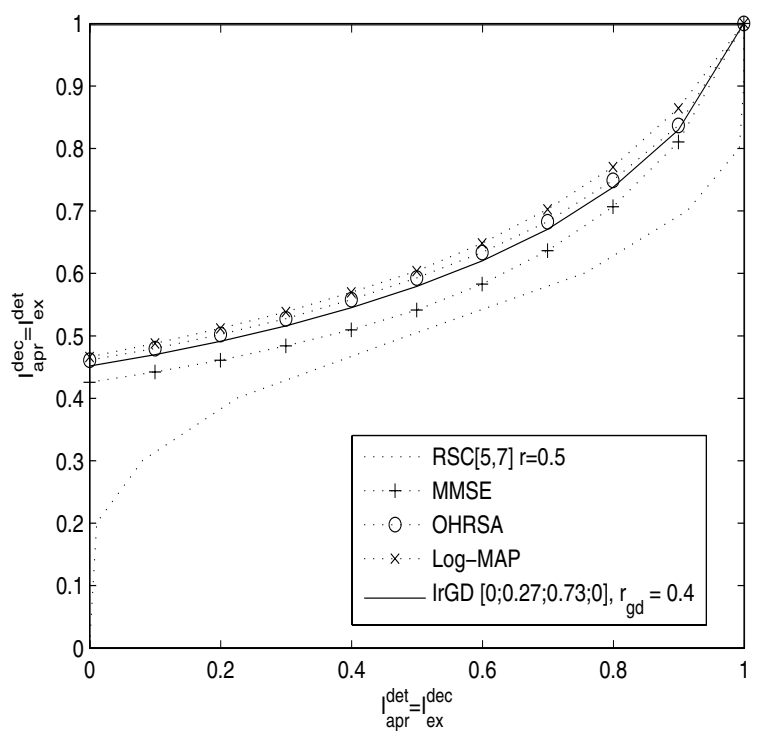

Fig. 4. EXIT chart analysis of the iterative DL-SDMA system invoking IrGD having a complexity ratio of $r_{g d}=0.4$ and a weighting-coefficient vector of $\boldsymbol{\alpha}=[0.27 ; 0.73 ; 0]$. The system operated at $E_{b} / N_{0}=1.7 \mathrm{~dB}$. A rate- 0.5 Recursive Systematic Convolutional (RSC) channel coder, having the octal generator polynomials of $G=[5,7]$, was employed for the system. The channel model was a flat-fading MIMO channel and the system parameters of Table II were used.

For further characterizing the complexity of the IrGD, in Figure 5 we illustrate the inner decoder's EXIT-curves for the IrGD aided iterative DL-SDMA system designed for different complexity ratios $r_{g d}$, ranging from $0.4,0.3,0.2$ to 0.19 . The weighting-coefficient vectors $\boldsymbol{\alpha}$ designed for each of the corresponding complexity ratios $r_{g d}$ are listed in Table III. As expected, by reducing the complexity ratio $r_{g d}$, the duty-cycles

of the lower-complexity detectors, such as the MMSE detector, becomes higher than those of the higher-complexity detectors, such as the OHRSA and Log-MAP schemes. On the other hand, as a consequence of reducing the complexity ratio $r_{g d}$, the EXITtunnel becomes narrower. The number of iterative decoding iterations required for attaining perfect convergence increases due to having a narrower EXIT-tunnel. Therefore, there is a tradeoff between the complexity ratio $r_{g d}$ corresponding to the singleiteration-complexity and the number of the decoding iterations $I_{g d}$. In order to reduce the overall complexity of the iterative DL-SDMA receiver, we have to take both effects into account. In Figure 6, we illustrated this trade-off. The required number of iterations $I_{g d}$ is increased, when reducing the complexity ratio $r_{g d}$. When $r_{g d}$ is lower than 0.18 , the EXIT-tunnel becomes narrow and the number of iterations required for navigating through the EXIT-tunnel becomes excessive.

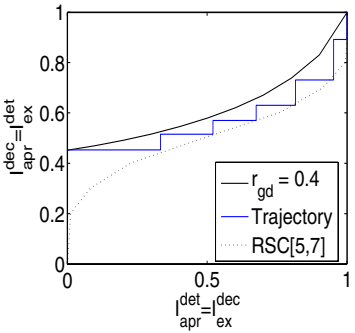

(a)

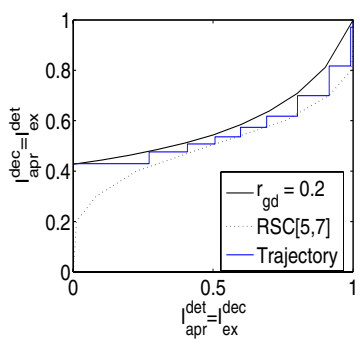

(c)

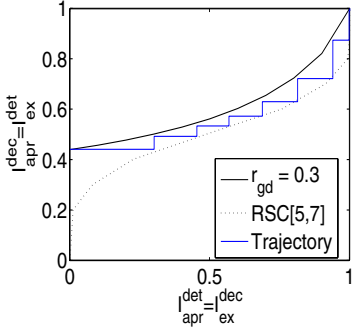

(b)

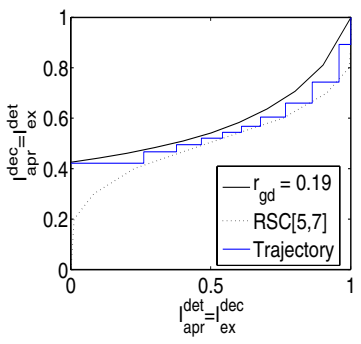

(d)

Fig. 5. EXIT chart analysis of the iterative DL-SDMA system invoking IrGD associated with different values of $r_{g d}$, ranging from $0.4,0.3,0.2,0.19$ in (a)(b)(c)(d), respectively. The systems operated at $E_{b} / N_{0}=1.7 d B$. A rate- 0.5 RSC [5,7] channel coder was employed for the system. The channel model was a flat-fading MIMO channel and the parameters of Table II were used.

Furthermore, based on Table III, we have generated Figure 


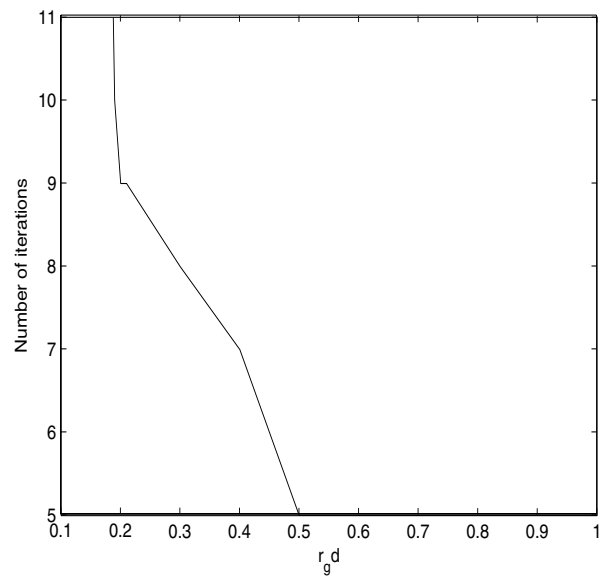

Fig. 6. The number of iterations required for perfect convergence of the IrGD aided iterative DL-SDMA systems associated with different complexity ratios $r_{g d}$ as recorded in Table III. The system operated at $E_{b} / N_{0}=1.7 \mathrm{~dB}$.

7, which illustrates the overall complexity of the IrGD aided iterative decoding process, i.e. $C_{g d, i t e r}$ and its complexity ratio, $r_{g d, i t e r}$, for different values of $r_{g d}$. We have found that the IrGD aided DL-SDMA system has the lowest iterative decoding complexity, when we have $r_{g d}=0.2$ and the corresponding weighting-coefficient vector is $\boldsymbol{\alpha}=[0.957 ; 0.043 ; 0]$ at $E_{b} / N_{0}$ $=1.7 \mathrm{~dB}$. The overall complexity ratio $r_{g d, \text { iter }}$ of the IrGD in the case of $r_{g d}=0.2$ is equal to 0.36 . In other words, it reduces the complexity by about $64 \%$ (1-0.36), compared to the Log-MAP detector aided iterative decoder. Compared to the OHRSA detector aided iterative decoder, the IrGD associated with $r_{g d}=0.2$ reduces the complexity by about $28 \%$ (1$806.40 / 1120.00)$

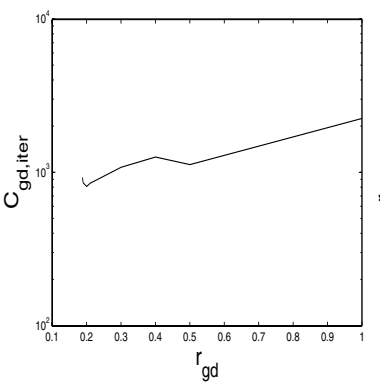

(a)

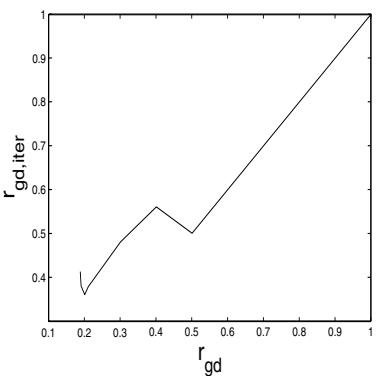

(b)
Fig. 7. Total complexity of the iterative IrGD in decoding process. The systems operated at $E_{b} / N_{0}=1.7 \mathrm{~dB}$.

\section{PERFORMANCE RESUltS}

In this section, we characterize the attainable performance of the IrGD aided iterative DL-SDMA system. The system parameters used are listed in Table II.

In Figure 8, we recorded the computational complexity of the iterative DL-SDMA system designed for reaching a target BER of $10^{-5}$. Observe in Figure 8 that the IrGD aided iterative DL-SDMA systems have a lower computational complexity than the benchmark systems. Observe that when the $E_{b} / N_{0}$ value is sufficiently high, such as $E_{b} / N_{0}=2.2 \mathrm{~dB}$, the IrGD aided system employed the MMSE detector during the entire iterativedecoding process. Therefore, the proposed IrGD provides a flexible detection framework. By switching amongst multiple

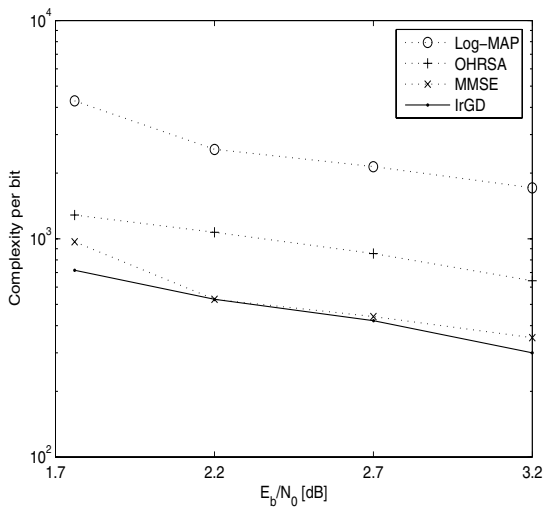

Fig. 8. Comparison of the computational complexity per detect bit for iterative decoding in the iterative DL-SDMA systems employing different detectors. The channel model was a flat fading MIMO channel and the parameters of Table II were used. The weighting-coefficient vector $\boldsymbol{\alpha}$ of the IrGD employed at the recorded $E_{b} / N_{0}$ from 1.7,2.2,2.7 to $3.2 \mathrm{~dB}$ are $[0.957 ; 0.043,0],[1 ; 0 ; 0],[0.9 ; 0.1 ; 0]$ and $[0.88 ; 0.12 ; 0]$, respectively.

detectors according to the appropriately designed weightingcoefficient vector $\boldsymbol{\alpha}$, the system may be expected to operate at the lowest possible iterative decoding complexity.

\section{CONCLUSIONS}

In this paper, we proposed the IrGD algorithm designed for reducing the complexity of the iterative decoding aided system. By appropriately designing the weighting-coefficient vector $\boldsymbol{\alpha}$, the IrGD aided system may potentially provide the lowest possible iterative decoding complexity. Quantitatively, the IrGD aided iterative DL-SDMA system associated with $\boldsymbol{\alpha}=[0.957 ; 0.043 ; 0]$ reduces the complexity by about $28 \%$, compared to the OHRSA aided benchmark systems.

\section{REFERENCES}

[1] B. Hochwald and S. ten Brink, "Achieving near-capacity on a multiple-antenna channel," IEEE Transactions on Communications, vol. 51, no. 3, pp. 389-399, March 2003.

[2] C.-Y. Wei, J. Akhtman, S. X. Ng, and L. Hanzo, "Iterative nearmaximum-likelihood detection in rank-deficient downlink SDMA systems," IEEE Transactions on Vehicular Technology, vol. 57, pp. $653-657$, Jan 2008.

[3] S. ten Brink, "Designing iterative decoding schemes with the extrinsic information transfer chart," $A E \ddot{U}$ International Journal of Electronics Communications, vol. 55, pp. 389 - 398, 2000.

[4] L. Hanzo and T. Keller, OFDM and MC-CDMA: A Primer. John Wiley and IEEE press, 2006.

[5] J. Akhtman, A. Wolfgang, S. Chen, and L. Hanzo, "An optimizedhierarchy-aided approximate log-MAP detector for MIMO systems," IEEE Transactions on Wireless Communications, vol. 6, no. 5, pp. 1900-1909, May 2007.

[6] L. Hanzo and M. Münster and B-J. Choi and T. Keller, OFDM and MC-CDMA for broadband multi-user communications, WLANs and Broadcasting. John Wiley and IEEE press, 2003.

[7] M. Tüchler and J. Hagenauer, "EXIT charts of irregular codes," in In Proceedings of Conference Information Sciences and Systems, Princeton [CDROM], NJ, USA, Mar, 2002.

[8] L.-U. Choi and R. Murch, "A transmit preprocessing technique for multiuser MIMO systems using a decomposition approach," IEEE Transactions on Wireless Communications, vol. 3, no. 1, pp. 20 24, 2004.

[9] D. Divsalar, S. Dolinar, and F. Pollara, "Serial turbo trellis coded modulation with rate-1 inner code," IEEE Global Telecommunications Conference, 2000. GLOBECOM 'O0., vol. 2, pp. 777 - 782, 2000 